gr-qc/9610020

TIT/HEP-346/COSMO-79

\title{
The Mass Shell of the Universe
}

\author{
A. Carlinif \\ Tokyo Institute of Technology, Oh-Okayama, Meguro-ku, Tokyo 152, Japan \\ J. Greensitt \\ Theoretical Physics Group, Ernest Orlando Lawrence Berkeley National Laboratory, \\ University of California, Berkeley, CA 94720 USA \\ and \\ Physics and Astronomy Dept., San Francisco State University, \\ San Francisco CA 94132, USA
}

\begin{abstract}
The classical field equations of general relativity can be expressed as a single geodesic equation, describing the free fall of a point particle in superspace. Based on this formulation, a "worldline" quantization of gravity, analogous to the FeynmanSchwinger treatment of particle propagation, is proposed, and a hidden mass-shell parameter is identified. We consider the effective action for the supermetric, which would be induced at one loop. In certain minisuperspace models, we find that this effective action is stationary for vanishing cosmological constant.
\end{abstract}

\footnotetext{
${ }^{1}$ E-mail: carlini@th.phys.titech.ac.jp

${ }^{2}$ E-mail: greensite@theorm.lbl.gov ; greensit@stars.sfsu.edu
} 


\section{Introduction}

In one of the classic papers of quantum electrodynamics, Feynman [1] suggested that relativistic electron propagation could be understood in terms of a sum over electron worldlines running both forwards and backwards in time. The evolution parameter was a path parameter, associated with the proper time of the electron worldlines, rather than the "clock time" of the laboratory. Related ideas were discussed by Stueckelberg, Fock, Nambu, and Schwinger [2]. In this article we would like to extend Feynman's worldline quantization of electron paths in spacetime to the quantization of a closed Universe propagating in superspace.

The elements of the proper-time approach for relativistic particles are, of course, very well-known. Consider for simplicity a spinless particle of mass $m$, propagating freely on a background spacetime with metric $g_{\mu \nu}$. The classical motion of the particle (i.e. the geodesic equation) is derived from variation of the worldline action

$$
\begin{aligned}
S_{p} & =-m \int d s \\
& =-m \int d \tau \sqrt{-g_{\mu \nu} \frac{d x^{\mu}}{d \tau} \frac{d x^{\nu}}{d \tau}}
\end{aligned}
$$

Removing the square-root by introduction of a Lagrange multiplier (lapse) $N$, we can write $S_{p}$ in the form

$$
S_{p}^{\prime}=m \int d \tau\left[\frac{1}{2 N} g_{\mu \nu} \frac{d x^{\mu}}{d \tau} \frac{d x^{\nu}}{d \tau}-\frac{1}{2} N\right]
$$

Applying the usual Legendre transform, one obtains the first-order form

$$
\begin{aligned}
S_{p}^{\prime \prime} & =\int d \tau\left[p_{\mu} \frac{d x^{\mu}}{d \tau}-N H\right] \\
H & =\frac{1}{2 m}\left(g^{\mu \nu} p_{\mu} p_{\nu}+m^{2}\right)
\end{aligned}
$$

Now go to the gauge $N=1$. In this gauge, $\tau=s$ is the proper-time parameter of the classical equations of motion. Adopting $s$ as the evolution parameter for the quantized theory, the amplitude for a relativistic spinless particle to propagate from point $x^{\prime \mu}$ to point $x^{\mu}$ in an interval $s$ can be expressed as a path-integral

$$
\begin{aligned}
G\left(x, x^{\prime} ; s\right) & =\int_{x^{\prime}}^{x} D x\left(s^{\prime}\right) \exp \left[\frac{i}{\hbar} \int_{0}^{s} L_{p}^{\prime} d s^{\prime}\right] \\
& =<x\left|e^{-i \mathcal{H}_{s} s / \hbar}\right| x^{\prime}> \\
L_{p}^{\prime} & =\frac{m}{2}\left(g_{\mu \nu} \frac{d x^{\mu}}{d s} \frac{d x^{\nu}}{d s}-1\right)
\end{aligned}
$$


Up to an operator-ordering, the corresponding Hamiltonian operator $\mathcal{H}_{s}$ describing state evolution in the evolution parameter $s$

$$
i \hbar \frac{\partial \psi}{\partial s}=\mathcal{H}_{s} \psi
$$

is obtained from the classical Hamiltonian (with $N=1$ ) via the usual replacement of c-number momenta by the corresponding operators, i.e.

$$
<x\left|\mathcal{H}_{s}\right| x^{\prime}>=\frac{1}{2 m}\left(-\hbar^{2} \square_{x}+m^{2}-i \epsilon+\xi R\right) \delta^{D}\left(x-x^{\prime}\right)\left[-g\left(x^{\prime}\right)\right]^{-1 / 2}
$$

The Feynman propagator is proportional to the inverse of $\mathcal{H}_{s}$, and the one-loop contribution to the gravitational effective action

$$
\mathcal{S}_{\text {eff }}\left[g_{\mu \nu}\right]=\frac{1}{2} i \hbar \operatorname{Tr} \ln \left[\mathcal{H}_{s}\right]
$$

is the trace logarithm of $\mathcal{H}_{s}$. The proper-time formalism itself has various uses, e.g. in calculating the Feynman propagator exactly in certain, especially simple, background electromagnetic fields, as well as in the evaluation of certain loop diagrams. We note that eigenvalues of the proper time Hamiltonian $\mathcal{H}_{s}$, such as those used in the evaluation of the effective action, can take on any value. Classically, however, the mass-shell condition $H=0$ is to be respected (this follows from variation of (3) by $N$ ), and for free particles this condition is imposed, in Dirac quantization, as a constraint on physical states $\mathcal{H}_{s} \psi=0$. For spinless particles, this operator constraint is just the Klein-Gordon equation in curved spacetime. In an interacting theory the mass-shell condition is relaxed somewhat; it is required only of asymptotic states. The 4-momentum of a virtual particle is allowed to violate the mass-shell condition.

\section{The Worldline Action of a Closed Universe}

We would now like to generalize the proper-time approach to the case of gravity in combination with any number of interacting bosonic fields; this calls for rewriting the gravitational action in the form

$$
S_{g}=-\mathcal{M} \int d s
$$

where $s$ is an invariant length parameter in the space of all fields modulo spatial diffeomorphisms, i.e. superspace, and $\mathcal{M}$ is an arbitrary dimensionless parameter. The only reasonable candidate for $s$ is the usual action of general relativity, so the problem 
is to reformulate that action as a proper time in superspace. Such a formulation was developed recently in ref. [3]; closely related ideas were put forward long ago in ref. [1]. The identification of action with proper time goes as follows:

Let $\left\{q^{a}(x), p_{a}(x)\right\}$ represent a set of gravitational and other bosonic fields, and their conjugate momenta, with the fields scaled by an appropriate power of $\kappa^{2}=$ $16 \pi G_{N}$ so as to be dimensionless. In a condensed notation, the standard ADM action has the form

$$
\begin{aligned}
S_{A D M} & =\int d^{4} x\left[p_{a} \partial_{0} q^{a}-N \mathcal{H}_{x}-N_{i} \mathcal{H}_{x}^{i}\right] \\
\mathcal{H}_{x} & =\kappa^{2} G^{a b} p_{a} p_{b}+\sqrt{g} U(q) \\
\mathcal{H}_{x}^{i} & =O^{i a}[q, \partial] p_{a}
\end{aligned}
$$

where $G^{a b}$ and $U$ are, respectively, the metric and potential in superspace, and the operator $O^{i a}$ is linear in the 3 -d spacetime covariant derivative. Go to the "shift gauge" $N_{i}=0$. The supermomentum constraints $\mathcal{H}^{i}=0$ are not lost by this choice, since they are still required for consistency of the Hamiltonian constraint with the equations of motion. Solving Hamilton's equation for the momenta in terms of velocities, then solving the Hamiltonian constraint for the lapse function $N$ in terms of velocities, and inserting both expressions into $S_{A D M}$, one obtains the Baierlein-Sharp-Wheeler (BSW) form of the gravitational action [5]

$$
S_{B S W}=-\int d^{4} x \sqrt{-\frac{1}{\kappa^{2}} \sqrt{g} U G_{a b} \partial_{0} q^{a} \partial_{0} q^{b}}
$$

in shift gauge $N_{i}=0$. The BSW action is to serve as a proper-time parameter. It is also useful to introduce an arbitrary mass-scale $\sigma$ in order to define an evolution parameter $t$ with dimensions of time, so that

$$
S_{B S W}=-\int d s=-\sigma \int d t
$$

Choose $x_{0}=t$. Comparing (11) with (10), we have

$$
d t=\frac{1}{\sigma} \int d^{3} x \sqrt{-\frac{1}{\kappa^{2}} \sqrt{g} U G_{a b} d q^{a} d q^{b}}
$$

Let $\tilde{N}$ denote the lapse function (derived by solving the Hamiltonian constraint) associated with the time parameter $t$

$$
\tilde{N}=\sqrt{-\frac{1}{4 \kappa^{2} \sqrt{g} U} G_{a b} \partial_{t} q^{a} \partial_{t} q^{b}}
$$

\footnotetext{
${ }^{3}$ Only the metric formulation will be considered here; hence the restriction to bosonic fields.
} 
Then we have

$$
1=-\frac{1}{\sigma} \int d^{3} x \frac{1}{2 \tilde{N} \kappa^{2}} G_{a b} \partial_{t} q^{a} \partial_{t} q^{b}
$$

Now $t$ denotes a "many-fingered" time variable, with the different possibilities distinguished by a choice of $\tilde{N}$. Equation (12) imposes only one global restriction on the choice of $\tilde{N}$. From eq. (13) we have

$$
\int d^{3} x \tilde{N} \sqrt{g} U=\int d^{3} x \sqrt{-\frac{1}{4 \kappa^{2}} \sqrt{g} U G_{a b} \partial_{t} q^{a} \partial_{t} q^{b}}
$$

which implies, from the definition (12), the condition

$$
\int d^{3} x \tilde{N} \sqrt{g} U=\frac{1}{2} \sigma
$$

For a given $\tilde{N}$ satisfying this condition, there corresponds a time variable proportional to $S_{B S W}$. The condition is solved trivially by

$$
\tilde{N}=\frac{\frac{1}{2} \sigma \mathcal{N}}{\int d^{3} x \mathcal{N} \sqrt{g} U}
$$

where $\mathcal{N}$ is unrestricted. Inserting this form for $\tilde{N}$ back into (14), we find

$$
1=-\frac{1}{\sigma^{2}} \int d^{3} x\left[\int d^{3} x^{\prime} \mathcal{N} \sqrt{g} U\right] \frac{1}{\mathcal{N} \kappa^{2}} G_{a b} \partial_{t} q^{a} \partial_{t} q^{b}
$$

or

$$
d s^{2}=-\int d^{3} x\left[\int d^{3} x^{\prime} \mathcal{N} \sqrt{g} U\right] \frac{1}{\mathcal{N} \kappa^{2}} G_{a b} d q^{a} d q^{b}
$$

Now introduce a mixed discrete/continuous "coordinate index" $(\alpha, x)$ in superspace:

$$
q^{(\alpha x)} \equiv q^{\alpha}(x)= \begin{cases}\mathcal{N}(x) & \alpha=0 \\ q^{a}(x) & \alpha=a \neq 0\end{cases}
$$

Apart from notation we are extending the definition of superspace slightly to include the non-dynamical field $\mathcal{N}(x)$, related via eq. (17) to the lapse parameter. Define a degenerate metric for this extended superspace

$$
\begin{aligned}
& \mathcal{G}_{(a x)(b y)}=\left[\int d^{3} x^{\prime} \mathcal{N} \sqrt{g} U\right] \frac{1}{\mathcal{N}(x) \kappa^{2}} G_{a b}(x) \delta^{3}(x-y) \\
& \mathcal{G}_{(0 x)(0 y)}=\mathcal{G}_{(a x)(0 y)}=\mathcal{G}_{(0 x)(b y)}=0
\end{aligned}
$$

With these definitions, and an obvious summation convention, eq. (19) becomes

$$
d s^{2}=-\mathcal{G}_{(\alpha x)(\beta y)} d q^{(\alpha x)} d q^{(\beta y)}
$$


The gravitational action then has the desired form

$$
\begin{aligned}
S_{g} & =-\mathcal{M} \int d s \\
& =-\mathcal{M} \int d \tau \sqrt{-\mathcal{G}_{(\alpha x)(\beta y)} \frac{d q^{(\alpha x)}}{d \tau} \frac{d q^{(\beta y)}}{d \tau}}
\end{aligned}
$$

Variation of the action $S_{g}$ w.r.t $q^{(\alpha x)}$ leads, in the usual way, to a geodesic equation

$$
\mathcal{G}_{(\alpha x)(\beta y)} \frac{d^{2} q^{(\beta y)}}{d s^{2}}+\frac{1}{2}\left(\frac{\delta \mathcal{G}_{(\alpha x)(\beta y)}}{\delta q^{(\gamma z)}}+\frac{\delta \mathcal{G}_{(\alpha x)(\gamma z)}}{\delta q^{(\beta y)}}-\frac{\delta \mathcal{G}_{(\beta y)(\gamma z)}}{\delta q^{(\alpha x)}}\right) \frac{d q^{(\beta y)}}{d s} \frac{d q^{(\gamma z)}}{d s}=0
$$

Identifying $d s=\sigma d t$, it is straightforward to verify that the $\alpha \neq 0$ components of (24) are the equations of motion

$$
\frac{\partial}{\partial t}\left[\frac{1}{2 \tilde{N} \kappa^{2}} G_{a b} \partial_{t} q^{b}\right]-\frac{1}{4 \tilde{N} \kappa^{2}} \frac{\partial G_{c d}}{\partial q^{a}} \partial_{t} q^{c} \partial_{t} q^{d}+\int d^{3} x^{\prime} \tilde{N} \frac{\delta}{\delta q^{a}(x)}(\sqrt{g} U)=0
$$

while the $\alpha=0$ component is the Hamiltonian constraint

$$
\frac{1}{4 \tilde{N}^{2} \kappa^{2}} G_{a b} \partial_{t} q^{a} \partial_{t} q^{b}+\sqrt{g} U=0
$$

These equations are identical to those obtained from the ADM action (9), with the gauge choice $N_{i}=0$ and $N=\tilde{N}$. We have therefore interpreted the classical field equations of general relativity as describing the free fall of a point particle in superspace.

Some further comments are in order. First, the choice of lapse $N=\tilde{N}$ imposes only one global condition (16) on the choice of lapse function. This does not result in any restriction on the choice of foliation, but only on the time-label $t$ associated with each hypersurface of a given foliation. A second point is that the degeneracy of the supermetric $\mathcal{G}_{(\alpha x)(\beta y)}$ in eq. (21) implies an infinite set of solutions for the geodesic between any two points in superspace. It is not hard to show that these solutions are related by (ordinary $\mathrm{D}=4$ ) time-reparametrizations, and have the same "proper time" interval in superspace (proportional to $S_{g}$ ) between those two points. Finally, let us note that the parameter $\mathcal{M}$ in $S_{g}$, which is analogous to the mass parameter $m$ in the relativistic particle action $S_{p}$, drops out of the classical configuration-space equations of motion.

Having recognized that the worldline action (23) leads to the same classical motion as the ADM action, we can proceed as in the relativistic particle case to derive the

\footnotetext{
${ }^{4}$ Eq. (23) can also be motivated from Jacobi's principle in mechanics, c.f. ref. [3].
} 
proper-time Hamiltonian. Again introducing a Lagrange multiplier $n$ to remove the square-root

$$
S_{g}^{\prime}=\mathcal{M} \int d \tau\left[\frac{1}{2 n} \mathcal{G}_{(a x)(b y)} \frac{d q^{(a x)}}{d \tau} \frac{d q^{(b y)}}{d \tau}-\frac{1}{2} n\right]
$$

the first-order form is

$$
\begin{aligned}
S_{g}^{\prime \prime} & =\int d \tau\left[p_{(a x)} \frac{d q^{(a x)}}{d \tau}-n H\right] \\
H & =\frac{1}{2 \mathcal{M}}\left[\mathcal{G}^{(a x)(b y)} p_{(a x)} p_{(b y)}+\mathcal{M}^{2}\right] \\
& =\frac{1}{2 \mathcal{M}}\left[\mathbb{E}+\mathcal{M}^{2}\right]
\end{aligned}
$$

where the supermetric

$$
\mathcal{G}^{(a x)(b y)} \doteq \frac{\mathcal{N} \kappa^{2} G^{a b} \delta^{3}(x-y)}{\int d^{3} x^{\prime} \mathcal{N} \sqrt{g} U}
$$

and the expression

$$
\begin{aligned}
\mathbb{E} & =\mathcal{G}^{(a x)(b y)} p_{(a x)} p_{(b y)} \\
& =\frac{\int d^{3} x \mathcal{N} \kappa^{2} G^{a b} p_{a} p_{b}}{\int d^{3} x^{\prime} \mathcal{N} \sqrt{g} U}
\end{aligned}
$$

were introduced in ref. [6]. Variation of (28) with respect to $q^{a}(x, \tau), p_{a}(x, \tau)$ and $\mathcal{N}(x, \tau), n(\tau)$ give us, respectively, the set of Hamiltonian equations and constraints

$$
\partial_{\tau} q^{a}(x)=\frac{n}{2 \mathcal{M}} \frac{\delta Æ}{\delta p_{a}(x)}, \quad \partial_{\tau} p_{a}(x)=-\frac{n}{2 \mathcal{M}} \frac{\delta Æ}{\delta q^{a}(x)}, \frac{\delta Æ}{\delta \mathcal{N}(x)}=0 \quad, \quad \rightleftarrows=-\mathcal{M}^{2}
$$

Setting $n=1$, so that $\tau=s=\sigma t$, these equations are equivalent to the usual Hamiltonian equations of motion and Hamiltonian constraint

$$
\begin{aligned}
\partial_{t} q^{a}(x) & =\int d^{3} x^{\prime} \tilde{N} \frac{\delta}{\delta p_{a}(x)} \mathcal{H} \\
\partial_{t} p_{a}(x) & =-\int d^{3} x^{\prime} \tilde{N} \frac{\delta}{\delta q^{a}(x)} \mathcal{H} \\
\mathcal{H} & =\frac{\kappa^{2}}{\mathcal{M}} G^{a b} p_{a} p_{b}+\mathcal{M} \sqrt{g} U=0
\end{aligned}
$$

in the $N=\tilde{N}, N_{i}=0$ gauge. These equations of motion and (Hamiltonian) constraint imply the remaining supermomentum constraint as a consistency condition.

The constant $\mathcal{M}$ is implicitly set to $\mathcal{M}=1$ in the usual Hamiltonian formulation of general relativity, but we note at this point that there is no overwhelming reason to 
make this choice. The constant $\mathcal{M}$ appears as a constant multiplicative factor in the worldline action (23), as does the mass $m$ in the worldline action (11). Both of these constants drop out of the corresponding geodesic equations. Just as there is no way of determining the mass of a particle from its trajectory in free fall, there is also no way of determining the value of $\mathcal{M}$ from a given solution of the configuration-space field equations. In the context of the first-order formulation, the condition $\Subset=-\mathcal{M}^{2}$ is in every sense analogous to the particle mass-shell condition $g^{\mu \nu} p_{\mu} p_{\nu}=-m^{2}$. It is therefore reasonable to identify $\mathcal{M}$ as a kind of (dimensionless) mass-shell parameter, and to dignify the constraint $\mathbb{E}=-\mathcal{M}^{2}$ with the title "mass-shell of the Universe".

\section{Quantization}

We now consider canonical quantization, in the "proper-time" gauge $n=1$. The corresponding Schrödinger equation is

$$
\begin{aligned}
i \hbar \frac{\partial \Psi}{\partial s} & =\mathcal{H}_{s} \Psi \\
& =\frac{1}{2 \mathcal{M}}\left(Æ+\mathcal{M}^{2}\right) \Psi
\end{aligned}
$$

which has the general $s$-dependent solution

$$
\begin{aligned}
\Psi[q, s] & =\sum_{\mathcal{E} \beta} a_{\mathcal{E} \beta} \Phi_{\mathcal{E} \beta}[q] e^{i\left(\mathcal{E}-\mathcal{M}^{2}\right) s /(2 \mathcal{M} \hbar)} \\
Æ \Phi_{\mathcal{E} \beta}[q] & =-\mathcal{E} \Phi_{\mathcal{E} \beta}[q]
\end{aligned}
$$

where the label $\beta$ distinguishes among a linearly independent set of eigenstates of $Æ$ with eigenvalue $-\mathcal{E}$. The classical constraint $\delta Æ / \delta \mathcal{N}=0$ becomes an operator

constraint $\frac{\delta \nsubseteq}{\delta \mathcal{N}} \Psi=0$. Inserting the eigenstate expansion (34), we find that each eigenstate $\Phi_{\mathcal{E}}$ satisfies a Wheeler-DeWitt equation

$$
\left[-\frac{\hbar^{2}}{\mathcal{E}} \kappa^{2 “} G^{a b} \frac{\delta^{2}}{\delta q^{a} \delta q^{b}}{ }^{\prime \prime}+\sqrt{g} U\right] \Phi_{\mathcal{E}}[q]=0
$$

associated with the parameter $\mathcal{E}$ (quotation marks indicate the ordering ambiguity). Finally, if we also impose the mass-shell constraint

$$
\rightleftarrows \Psi=-\mathcal{M}^{2} \Psi
$$

then the only physical states are those with $\mathcal{E}=\mathcal{M}^{2}$, and the (classically indeterminate) constant $\mathcal{M}$ can be absorbed, via

$$
\hbar_{e f f}=\frac{\hbar}{\mathcal{M}}
$$


into a rescaling of Planck's constant.

There are two ways in which the off mass-shell states, with $\mathcal{E} \neq \mathcal{M}^{2}$, may be physically relevant. First, the mass-shell constraint (36) may not really be a constraint, at the quantum level. The mass-shell condition is derived by trading the square-root form of the action for an expression involving a Lagrange multiplier. What if one avoids this step, and quantizes the square-root action $S_{B S W}$ directly? This approach has been advocated in ref. [6, 7], and it leads to a formulation in which the dynamical equation (33) is supplemented by the constraints $(\delta Æ / \delta \mathcal{N}) \Psi=0$, but without the mass-shell constraint $\mathbb{E}=-\mathcal{M}^{2}$. It should be noted, once again, that there is no way to determine $\mathcal{M}$ classically, or to verify the mass-shell condition $Æ=-\mathcal{M}^{2}$, since the configuration-space equations are independent of $\mathcal{M}^{2}$. Determination of $\mathcal{M}^{2}$ would require a violation of the Einstein field equations; it is analogous to trying to determine the mass of a particle from its trajectory in free fall. Moreover, the freedom to choose arbitrary foliations of 4-space is already reflected in the constraint $(\delta Æ / \delta \mathcal{N}) \Psi=0$. In the formulation of [6, 7], the physical Hilbert space is spanned by the solutions of a family of Wheeler-DeWitt equations (35), one equation for each eigenvalue $-\mathcal{E}$ of $Æ$.

The second way in which off mass-shell states could become relevant is suggested by the phenomenon of black hole evaporation. Although it is known that black holes must lose mass via Hawking radiation, it is not known what the final state of the radiative process will be. It is possible that the black hole disappears entirely, and this might be considered a case of topology change involving the production of a "baby universe", analogous to similar processes in string theory. It is also possible that the evaporation is not complete, and the black hole leaves a remnant. Let us suppose that the first alternative, namely, complete evaporation accompanied by baby universe production, is the correct one. In that case the Universe is not really in free fall; there will be interactions associated with topology changing processes (emission and absorbtion of baby universes).

A satisfactory description of topology-changing processes awaits development of a "third-quantized" theory of gravity [8]; unfortunately, at present, we do not even have a satisfactory understanding of second-quantized gravity. Still, it may be possible to obtain some insight into "multi-versal" effects via the worldline formulation. For example, by direct analogy to eq. (7), the 1-loop contribution of virtual universe 
loops to the effective action would be

$$
S_{e f f}\left[\mathcal{G}_{a b}\right]=\frac{i \hbar}{2} \operatorname{Tr} \ln \left[\mathbb{E}+\mathcal{M}^{2}\right]
$$

where the trace runs over a basis of states $\Phi_{\mathcal{E}}$ satisfying the one-parameter family of Wheeler-DeWitt equations (35). Of course, the supermetric $\mathcal{G}_{a b}$, unlike the ordinary spacetime metric $g_{\mu \nu}$, is not arbitrary; it is constrained to have the form (21). Therefore $S_{\text {eff }}$ may be regarded as a functional of the potential term $U(q)$. But the form of $U(q)$ is also tightly constrained: it is the sum of all possible potential terms that could appear in an ADM Hamiltonian. With this restriction, $S_{\text {eff }}$ is just a function of the coupling constants of each possible interaction term, i.e.

$$
S_{e f f}\left[\mathcal{G}_{a b}\right]=S_{e f f}\left[\lambda, e^{2}, g^{2}, \ldots\right]
$$

and the couplings are now viewed as dynamical variables. Variation of $S_{\text {eff }}$ with respect to the couplings could, in principle, determine their phenomenological values, very much in the spirit of Coleman's "Big Fix" [9].

Let us illustrate this possibility with a minisuperspace toy model, in which the supermetric $\mathcal{G}_{a b}$ depends on one parameter only, namely, the cosmological constant $\lambda$. The starting point is the minisuperspace action representing a closed, homogenous and isotropic Friedman-Robertson-Walker (FRW) universe filled with a three-component, minimally coupled scalar field $\vec{\phi} \doteq\left(\phi_{1}, \phi_{2}, \phi_{3}\right)$, i.e.

$$
S=\frac{1}{2} \int d t\left[-\frac{a \dot{a}^{2}}{N}+\frac{a^{3} \dot{\vec{\phi}} \cdot \dot{\vec{\phi}}}{N}+N\left(a-\lambda a^{3}\right)\right]
$$

where the 4-d invariant length is

$$
d s^{2}=\hat{\sigma}^{2}\left(-N^{2} d t^{2}+a^{2} d \Omega_{3}^{2}\right)
$$

and with $\hat{\sigma}^{2} \doteq 2 G_{N} / 3 \pi$.

With the choice of coordinates $q^{0}=a, q^{i}=\phi^{i}$, the supermetric for the corresponding worldline action

$$
S_{g}=-\mathcal{M} \int d \tau \sqrt{-\mathcal{G}_{a b} \dot{q}^{a} \dot{q}^{b}}
$$

reads

$$
\mathcal{G}_{i i}=-a^{2} \mathcal{G}_{00}=a^{4}\left(\lambda a^{2}-1\right) \quad ; \quad i=1,2,3
$$


Now, on general grounds of diffeomorphism invariance in minisuperspace, the effective action for a generic FRW universe will have a weak-curvature (adiabatic) expansion of the form

$$
S_{e f f}\left[\mathcal{G}_{a b}\right]=\int d a \int d \vec{\phi} \sqrt{|\mathcal{G}|}\left[\Lambda_{S}+\kappa_{S} \mathcal{R}+O\left(\mathcal{R}^{2}\right)\right]
$$

where $\Lambda_{S}$ and $\kappa_{S}$ are the (dimensionless) "supercosmological constant" and "super Newton's constant", respectively, and $\mathcal{R}$ is the scalar "supercurvature" of $\mathcal{G}_{a b}$. In general, since $\Lambda_{S}, \kappa_{S}$ are divergent at one loop, even in simple minisuperspace models, it must be assumed that either these constants are renormalized (and there exists a bare action $S_{0}\left[\mathcal{G}_{a b}\right]$ ), or else that there is a fundamental cutoff of some kind in the theory.

Let us now temporarily compactify the ranges of integration in (44) so that the scale factor runs from $a=0$ to $a=\bar{a}$ and the scalar fields run from $\phi_{i}=-\phi_{i M}$ to $\phi_{i}=\phi_{i M}$, and keep only the leading term in the adiabatic expansion (44). Then the effective action (44) reads

$$
\begin{aligned}
S_{e f f} & \simeq \Lambda_{S} \int_{0}^{\bar{a}} d a \int_{-\phi_{1 M}}^{\phi_{1 M}} d \phi_{1} \int_{-\phi_{2 M}}^{\phi_{2 M}} d \phi_{2} \int_{-\phi_{3 M}}^{\phi_{3 M}} d \phi_{3} a^{7}\left(\lambda a^{2}-1\right)^{2} \\
& =\left(\int d^{3} \phi\right) \Lambda_{S} \bar{a}^{8}\left(\frac{\lambda^{2} \bar{a}^{4}}{12}-\frac{\lambda \bar{a}^{2}}{5}+\frac{1}{8}\right)
\end{aligned}
$$

It is easy to check that $S_{\text {eff }}$ is stationary at

$$
\frac{d S_{e f f}}{d \lambda}=0 \quad \Longrightarrow \quad \lambda \simeq \frac{6}{5 \bar{a}^{2}}
$$

with the result that $\lambda \rightarrow 0^{+}$as $\bar{a} \rightarrow \infty$. It is also straightforward to show that this stationary point is actually a minimum for $S_{\text {eff }}$ provided that $\Lambda_{S}>0$.

\section{Inclusion of Mass Terms and Supercurvature}

Any minisuperspace model is a toy, and only illustrates effects which might (or might not) be present in the full theory. Still, even within the category of toy models, it is interesting to study whether the vanishing of the cosmological constant survives some modest complications of the minisuperspace action, and/or improvements in the approximations for (44), e.g., the inclusion of contributions from the supercurvature terms in the adiabatic expansion of the effective action. 
We consider the action for a FRW universe filled with $N_{s}$ scalar fields $\left(\phi_{1}, \ldots, \phi_{N_{s}}\right) \doteq \vec{\phi}$ with potential $V(\phi)$, i.e.

$$
S=\frac{1}{2} \int d t\left\{-\frac{a \dot{a}^{2}}{N}+\frac{a^{3} \dot{\vec{\phi}} \cdot \dot{\vec{\phi}}}{N}+N a\left[1-(\lambda+V(\phi)) a^{2}\right]\right\}
$$

Again choosing coordinates $q^{0}=a, q^{i}=\phi^{i}$, then, from eq. (42), the diagonal, $N_{s}+1$-dimensional worldline supermetric $\mathcal{G}_{a b}$ is just

$$
\mathcal{G}_{i i}=-a^{2} \mathcal{G}_{00}=a^{4}\left[(\lambda+V(\phi)) a^{2}-1\right] \quad ; \quad i=1, \ldots N_{s}
$$

and the effective action is given by eq. (44). The question is whether the stationary point of the effective action (44), with supermetric (48), is still at $\lambda=0^{+}$. We will now consider some cases for various numbers of scalar fields, with and without mass-term potentials.

\section{1 $N_{s}$ massless scalar fields}

As a first example, we consider the model of a FRW universe filled with $N_{s}$ massless, minimally coupled scalar fields, i.e. the case with potential

$$
V(\phi)=0
$$

For this scalar potential, inserting the supermetric (48) into eq. (44) we can easily write down for the effective action (neglecting the 'supercurvature' contributions)

$$
\begin{aligned}
S_{e f f} & \simeq \Lambda_{S} \int_{0}^{\bar{a}} d a \int_{-\phi_{1 M}}^{\phi_{1 M}} d \phi_{1} \cdots \int_{-\phi_{N_{s} M}}^{\phi_{N_{s} M}} d \phi_{N_{s}} a^{2 N_{s}+1}\left|\lambda a^{2}-1\right|^{\left(N_{s}+1\right) / 2} \\
& =\frac{N_{s} ! \Lambda_{S}\left(\prod_{i=1}^{N_{s}} I_{\phi_{i}}\right) \bar{a}^{2\left(N_{s}+1\right)}}{3\left(3 N_{s}+1\right) ! ! x^{N_{s}+1}}\left\{2^{N_{s}}\left(N_{s}-1\right) ! !+[\Theta(x-1)\right. \\
& \left.-\Theta(1-x)] x^{N_{s}}|x-1|^{\left(N_{s}+3\right) / 2} \sum_{k=0}^{N_{s}} 2^{k} \frac{\left(3 N_{s}-2 k+1\right) ! !}{\left(N_{s}-k\right) !} x^{-k}\right\}
\end{aligned}
$$

where $\Theta(x)$ is the Heaviside step function and the quantities $x$ and $I_{\phi_{i}}$ are given by

$$
x \doteq \lambda \bar{a}^{2}
$$

and

$$
I_{\phi_{i}} \doteq \int_{-\phi_{i M}}^{\phi_{i M}} d \phi_{i}
$$


Taking the derivative of the effective action (50) with respect to $\lambda$ we get

$$
\begin{aligned}
\frac{d S_{e f f}}{d \lambda} & =\frac{2^{N_{s}}\left(N_{s}+1\right) ! \Lambda_{S}\left(\prod_{i=1}^{N_{s}} I_{\phi_{i}}\right) \bar{a}^{2\left(N_{s}+2\right)}}{3\left(3 N_{s}+1\right) ! ! x^{N_{s}+2}}\left\{-\left(N_{s}-1\right) ! !\right. \\
& \left.+|x-1|^{\left(N_{s}+1\right) / 2} \sum_{k=0}^{N_{s}+1} 2^{-k} \frac{\left(N_{s}+2 k-1\right) ! !}{k !} x^{k}\right\}
\end{aligned}
$$

Unfortunately, the stationarity condition coming from eq. (53), i.e. by imposing $d S_{\text {eff }} / d \lambda=0$, cannot be easily solved for arbitrary $N_{s}$. However, one can still prove the existence of a finite number (at least one) of stationary points of $S_{\text {eff }}$ which are all at $x>0$ and at a finite distance from the origin $x=0$. In fact, studying the behaviour of $d S_{\text {eff }} / d \lambda$ in the range $x \geq 1$, we get (for $\Lambda_{S}>0$ )

$$
\begin{aligned}
\frac{d S_{\text {eff }}}{d \lambda}(x=1) & =-\frac{2^{N_{s}}\left(N_{s}+1\right) ! ! \Lambda_{S}\left(\prod_{i=1}^{N_{s}} I_{\phi_{i}}\right) \bar{a}^{2\left(N_{s}+2\right)}}{3\left(3 N_{s}+1\right) ! !}<0 \\
\frac{d S_{\text {eff }}}{d \lambda}(x \rightarrow+\infty) & \sim \frac{\Lambda_{S}\left(\prod_{i=1}^{N_{s}} I_{\phi_{i}}\right) \bar{a}^{2\left(N_{s}+2\right)} x^{\left(N_{s}-1\right) / 2}}{6}>0
\end{aligned}
$$

(with the inequality signs reversed in the case $\Lambda_{S}<0$ ). On the other hand, it is possible to check (i.e., by using Mathematica), that

$$
\frac{d S_{e f f}}{d \lambda}<0 \quad ; \quad \forall x \leq 0
$$

(again with the inequality sign reversed in the case $\Lambda_{S}<0$ ). In other words, eqs. (54) and (55) imply that $d S_{\text {eff }} / d \lambda$ will have at least one finite zero at $x \doteq x_{1}>1$, and at most a finite number of extra zeros at $x \doteq x_{n}=$ finite $>0$. Therefore, the effective action $S_{\text {eff }}$ will be stationary at

$$
\left.\frac{d S_{e f f}}{d \lambda}\right|_{x_{1}}=0 \quad \Longrightarrow \lambda=\frac{x_{1}}{\bar{a}^{2}}
$$

(or, at any other of the points $x_{n}=c_{n} x_{1}$, with $c_{n}=$ constant $>0$ ). Removing the cutoff, $\bar{a} \rightarrow \infty$, this leads again to the result that $\lambda=0^{+}$.

$N_{s}=0$ scalar fields In this case the minisuperspace is one-dimensional, with the single metric component

$$
\mathcal{G}_{00}=-a^{2}\left(\lambda a^{2}-1\right)
$$


The supercurvature $\mathcal{R}$ is, of course, identically zero. One can then immediately write the effective action from eq. (44) as

$$
\begin{aligned}
S_{e f f}[\lambda] & =\Lambda_{S} \int_{0}^{\bar{a}} d a a\left|\lambda a^{2}-1\right|^{1 / 2} \\
& =\frac{\Lambda_{S} \bar{a}^{2}}{3 x}\left\{1+[\Theta(x-1)-\Theta(1-x)]|x-1|^{3 / 2}\right\}
\end{aligned}
$$

Taking the derivative of (58) with respect to $\lambda$, one obtains

$$
\frac{d S_{\text {eff }}}{d \lambda}=0 \Longrightarrow \lambda=\frac{\left[(3+2 \sqrt{2})^{1 / 3}+(3-2 \sqrt{2})^{1 / 3}-1\right]}{\bar{a}^{2}}
$$

with the result that $\lambda \rightarrow 0^{+}$as the regulator $\bar{a}$ is removed. It is also straightforward to check that this stationary point is a minimum for the effective action if $\Lambda_{S}>0$.

$N_{s}=1$ massless scalar field (with supercurvature contribution) Next we consider the case of a single massless scalar field. In this case the supermetric will have two independent diagonal entries, $\mathcal{G}_{00}$ and $\mathcal{G}_{11}$, which can again be read from eq. (48), and we can also improve the evaluation of the effective action by including the contribution of the supercurvature term given by

$$
\mathcal{R}=-\frac{4 \lambda}{a^{2}\left(\lambda a^{2}-1\right)^{3}}
$$

The effective action, up to first order contributions from the adiabatic expansion in $\mathcal{R}$, reads

$$
\begin{aligned}
S_{e f f} & \simeq \int_{0}^{\bar{a}} d a \int_{-\phi_{M}}^{\phi_{M}} d \phi\left[\Lambda_{S} a^{3}\left|\lambda a^{2}-1\right|-4 \kappa_{S} \frac{\lambda a\left|\lambda a^{2}-1\right|}{\left(\lambda a^{2}-1\right)^{3}}\right] \\
& =I_{\phi}\left\{\Lambda_{S} \frac{\bar{a}^{4}}{6}\left[\left(x-\frac{3}{2}+\frac{1}{x^{2}}\right) \Theta(x-1)+\left(\frac{3}{2}-x\right) \Theta(1-x)\right]\right. \\
& \left.-\frac{\kappa_{S}}{(x-1)}[(x-3) \Theta(x-1)+2 x \Theta(1-x)]\right\}
\end{aligned}
$$

Evaluating the derivative of (61) with respect to $\lambda$ we get

$$
\begin{aligned}
\frac{d S_{e f f}}{d \lambda} & \simeq \frac{I_{\phi} \Lambda_{S} \bar{a}^{6}}{6}\left\{\left[\frac{\left(x^{3}-2\right)}{x^{3}}-\frac{\alpha}{(x-1)^{2}}\right] \Theta(x-1)\right. \\
& \left.-\left[1-\frac{\alpha}{(x-1)^{2}}\right] \Theta(1-x)\right\}
\end{aligned}
$$

where we have introduced the quantity

$$
\alpha \doteq \frac{12 \kappa_{S}}{\Lambda_{S} \bar{a}^{4}}
$$


Now, provided that $\Lambda_{S} \neq 0$, [ imposing the stationarity condition with $d S_{\text {eff }} / d \lambda$ given by eq. (62), and noting from eq. (63) that the contribution coming from the supercurvature term can be neglected in the limit when the regulator for the scale factor is removed $(\bar{a} \rightarrow \infty)$, it is straightforward to get the result

$$
\frac{d S_{e f f}}{d \lambda}=0 \quad \Longrightarrow \quad \lambda \simeq \frac{2^{1 / 3}}{\bar{a}^{2}}
$$

In other words, the effective action is stationary at $\lambda=0^{+}$as the regulator $\bar{a} \rightarrow \infty$ is removed. Moreover, evaluating the second order derivative of $S_{\text {eff }}$ with respect to $\lambda$ it is easy to see that the stationary point is a minimum for $S_{\text {eff }}$ if $\Lambda_{S}>0$.

$N_{s}=3$ massless scalar fields (with supercurvature contribution) The analysis of the model of a FRW universe filled with three massless scalar fields essentially proceeds along the same lines as in the previous paragraph. In particular, the supermetric now has two extra diagonal elements (i.e., $\mathcal{G}_{22}=\mathcal{G}_{33} \equiv \mathcal{G}_{11}$, plus $\mathcal{G}_{00}$, all readable from eq. (48)), and the supercurvature turns out as

$$
\mathcal{R}=\frac{6\left[9\left(\lambda a^{2}\right)^{2}-14 \lambda a^{2}+4\right]}{a^{4}\left(\lambda a^{2}-1\right)^{3}}
$$

so that the effective action, up to first order contributions from $\mathcal{R}$, reads

$$
\begin{aligned}
S_{e f f} & \simeq \int_{0}^{\bar{a}} d a \int_{-\phi_{1 M}}^{\phi_{1 M}} d \phi_{1} \int_{-\phi_{2 M}}^{\phi_{2 M}} d \phi_{2} \int_{-\phi_{3 M}}^{\phi_{3 M}} d \phi_{3}\left\{\Lambda_{S} a^{7}\left(\lambda a^{2}-1\right)^{2}\right. \\
& \left.+6 \kappa_{S} \frac{a^{3}\left[9\left(\lambda a^{2}\right)^{2}-14 \lambda a^{2}+4\right]}{\left(\lambda a^{2}-1\right)}\right\} \\
& =\left(\prod_{i=1}^{3} I_{\phi_{i}}\right) \bar{a}^{4}\left[\Lambda_{S} \bar{a}^{4}\left(\frac{x^{2}}{12}-\frac{x}{5}+\frac{1}{8}\right)\right. \\
& \left.+3 \frac{\kappa_{S}}{x^{2}}\left(3 x^{3}-\frac{5}{2} x^{2}-x-\ln |x-1|\right)\right]
\end{aligned}
$$

Finally, taking the derivative with respect to $\lambda$ we get

$$
\begin{aligned}
\frac{d S_{e f f}}{d \lambda} & \simeq\left(\prod_{i=1}^{3} I_{\phi_{i}}\right) \Lambda_{S} \bar{a}^{10}\left\{\frac{x}{6}-\frac{1}{5}\right. \\
& \left.+\frac{3}{4} \alpha\left[1+\frac{(x-2)}{3 x^{2}(x-1)}+\frac{2}{3 x^{3}} \ln |x-1|\right]\right\}
\end{aligned}
$$

\footnotetext{
${ }^{5}$ When $\Lambda_{S}=0$ the analysis of the stationary points of $S_{\text {eff }}$ critically depends on the relative scaling between $\lambda$ and the cutoff $\bar{a}$, and is not conclusive.
} 
where $\alpha$ is again defined according to eq. (63). Let us consider the case $\Lambda_{S} \neq 0$ first. In this ansatz, using similar arguments to those of the previous section one can easily see that the contribution coming from the supercurvature term (the $\alpha$ term in eq. (67)) can be neglected when removing the cutoff $\bar{a}$, and therefore the stationarity condition for $S_{e f f}$ implied by eq. (67) becomes

$$
\frac{d S_{\text {eff }}}{d \lambda}=0 \quad \Longrightarrow \quad \lambda \simeq \frac{6}{5 \bar{a}^{2}}
$$

Eq. (68) again predicts the value $\lambda=0^{+}$as $\bar{a} \rightarrow \infty$. Contrarily to the previous model for a single scalar field, in the three massless scalar field case we can also consider the ansatz $\Lambda_{S}=0$. In this case, in fact, it is easy to check from eq. (67) that the stationarity condition for $S_{\text {eff }}$ has a solution at the point $x \dot{=} x_{2}=$ finite $>0$

$$
\left.\frac{d S_{\text {eff }}}{d \lambda}\right|_{x_{2}}=0 \Longrightarrow \lambda \simeq \frac{x_{2}}{\bar{a}^{2}}
$$

In other words, also in this case $\lambda=0^{+}$is a stationary point for the effective action. Finally, evaluating the second order derivative of $S_{\text {eff }}$ with respect to $\lambda$, at the stationary points (68) or (69), it is easy to check that these are minima for $S_{\text {eff }}$ either if $\Lambda_{S}>0$ (for any $\kappa_{S}$ ) or if $\kappa_{S}>0\left(\right.$ when $\left.\Lambda_{S}=0\right)$.

\section{2 $N_{s}=4 r-1$ massive scalar fields}

The next complication of the FRW universe toy model is to consider the case of an odd number $N_{s}=4 r-1(r=1,2, .$.$) of massive, minimally coupled scalar fields (the$ case of one single massive scalar field is separately treated in the Appendix) with potential

$$
V(\phi)=\sum_{i=1}^{4 r-1} m_{i}^{2} \phi_{i}^{2}
$$

The supermetric is once again diagonal, with $4 r-1$ identical entries $\mathcal{G}_{i i}$ plus $\mathcal{G}_{00}$ (which can be easily read off eq. (48)), and there is no ambiguity in the sign of its determinant when evaluating $S_{\text {eff }}$. In particular, making use of the binomial expansion theorem three times, the effective action can be written (neglecting the 'supercurvature' contributions) as

$$
\begin{aligned}
S_{e f f} & \simeq \Lambda_{S} \int_{0}^{\bar{a}} d a \int_{-\phi_{1 M}}^{\phi_{1 M}} d \phi_{1} \cdot . \int_{-\phi_{N_{s} M}}^{\phi_{N_{s} M}} d \phi_{N_{s}} a^{2 N_{s}+1}\left[\left(\sum_{i=1}^{N_{s}} m_{i}^{2} \phi_{i}^{2}+\lambda\right) a^{2}-1\right]^{2 r} \\
& =\frac{1}{2}\left(\frac{N_{s}+1}{2}\right) ! \Lambda_{S}\left(\prod_{i=1}^{N_{s}} I_{\phi_{i}}\right) \bar{a}^{2\left(N_{s}+1\right)}
\end{aligned}
$$




$$
\times \sum_{k=0}^{2 r} \sum_{j=0}^{k} \sum_{s_{1} . . s_{N_{s}}=0}^{k-j} \frac{\delta\left(\sum_{i=1}^{N_{s}} s_{i}-k+j\right)(-1)^{k} x^{j} y_{1}^{s_{1}} \ldots y_{N_{s}}^{s_{N_{s}}}}{\left(2 s_{1}+1\right) s_{1} ! \ldots\left(2 s_{N_{s}}+1\right) s_{N_{s}} !(2 r-k) ! j !(4 r+k)}
$$

where we have used the 'cosmological constant-variable' defined by eq. (51) and introduced the new 'mass-variable' $y_{i}$ according to

$$
y_{i} \doteq m_{i}^{2} \phi_{i M}^{2} \bar{a}^{2}
$$

Now, in order to find the stationary points of $S_{\text {eff }}$, we can simplify the whole analysis by taking partial derivatives with respect to $\lambda$ and $m_{i}^{2}$ and evaluating them at $y_{i}=0$ (i.e., at zero masses for the scalar fields $\phi_{i}$ ). Proceeding in this way and noting that the only relevant terms surviving at $y_{i}=0$ from the sums in eq. (71) are, for the derivative with respect to $m_{i}^{2}$, those with $j=k-1$, and, for the derivative with respect to $\lambda$ and the effective action itself, those with $j=k$, we obtain the formulas

$$
\begin{aligned}
\left.\frac{\partial S_{e f f}}{\partial\left(m_{i}^{2}\right)}\right|_{y_{i}=0} & =\left.\frac{\phi_{i M}^{2}}{3} \frac{\partial S_{e f f}}{\partial \lambda}\right|_{y_{i}=0}=\frac{\phi_{i M}^{2}}{3} \frac{\partial}{\partial \lambda}\left[\left.S_{\text {eff }}\right|_{y_{i}=0}\right] \\
& \simeq \frac{\Lambda_{S} \bar{a}^{2(4 r+1)}\left(I_{\phi_{i}}\right)^{2}\left(\prod_{j=1}^{4 r-1} I_{\phi_{j}}\right)}{24} \\
& \times \sum_{k=0}^{2 r}(-1)^{k}\left(\begin{array}{c}
2 r \\
k
\end{array}\right) \frac{k}{(4 r+k)} x^{k-1}
\end{aligned}
$$

The effective action (71) evaluated at $y_{i}=0$ is, of course, the same as that considered in section 4.1 for the case of $N_{s}$ massless scalar fields (with the restriction that $N_{s}=4 r-1$ ), and as a consequence also the stationarity conditions derived from eqs. (73) are equivalent to the massless model condition coming from eq. (53). Then the result is that also for the massive model considered here there is at least one (trivial) stationary point at $m_{i}=0(i=1,2, . .4 r-1)$ and $\lambda$ given by eq. (56).

Moreover, since the general stationarity conditions which one would derive by equating the partial derivatives of $S_{\text {eff }}$, eq. (71), with respect to $\lambda$ and $m_{i}^{2}$ for arbitrary $m_{i}$ are still polynomial equations of finite order in $x$ and $y_{i}$, it is easy to see that any other eventual stationary point for the effective action would still be at $\left|x_{n}\right|=$ finite,$\left|y_{i, n}\right|=$ finite. Therefore, we can again conclude that the stationary point for the effective action representing a FRW universe filled with $N_{s}=4 r-1$ massive scalar fields is, after removal of the cutoffs, unique, i.e. at $|\lambda|=0$ and $m_{i}=0$ $(i=1,2, . .4 r-1) \cdot$.

\footnotetext{
${ }^{6}$ The modulus in the value for $\lambda$ is actually due to our ignorance about the signs of the other eventual stationary points $x_{n}$ and $y_{i, n}$.
} 
$N_{s}=3$ massive scalar fields In the case $N_{s}=3$, the algebra is especially simple. In this case the effective action (71) simplifies to

$$
\begin{aligned}
S_{\text {eff }} & =\Lambda_{S} \bar{a}^{8}\left(\prod_{i=1}^{3} I_{\phi_{i}}\right)\left[\frac { 1 } { 1 2 } \left(\frac{1}{5} \sum_{i=1}^{3} y_{i}^{2}+\frac{2}{9} \sum_{i>j=1}^{3} y_{i} y_{j}\right.\right. \\
& \left.\left.+\frac{2}{3} x \sum_{i=1}^{3} y_{i}+x^{2}\right)-\frac{1}{5}\left(\frac{1}{3} \sum_{i=1}^{3} y_{i}+x\right)+\frac{1}{8}\right]
\end{aligned}
$$

In particular, the partial derivatives with respect to $\lambda$ and $m_{i}^{2}$ turn out as

$$
\begin{gathered}
\frac{\partial S_{\text {eff }}}{\partial\left(m_{i}^{2}\right)}=\frac{\Lambda_{S} \bar{a}^{10}\left(\prod_{j=1}^{3} I_{\phi_{j}}\right)\left(I_{\phi_{i}}\right)^{2}}{120}\left[y_{i}+\frac{5}{9} \sum_{j \neq i} y_{j}+5 \frac{x}{3}-2\right] \\
\frac{\partial S_{e f f}}{\partial \lambda}=\frac{\Lambda_{S} \bar{a}^{10}\left(\prod_{i=1}^{3} I_{\phi_{i}}\right)}{6}\left[\frac{1}{3} \sum_{i=1}^{3} y_{i}+x-\frac{6}{5}\right]
\end{gathered}
$$

from which it is straightforward to find that the unique stationary point of $S_{\text {eff }}$ is at

$$
\begin{aligned}
\frac{\partial S_{\text {eff }}}{\partial \lambda}=0 & \Longrightarrow \lambda=\frac{6}{5 \bar{a}^{2}} \\
\frac{\partial S_{\text {eff }}}{\partial\left(m_{i}^{2}\right)}=0 & \Longrightarrow m_{i}^{2}=0 \quad ; \quad i=1,2,3
\end{aligned}
$$

On removing the cutoffs we find, as anticipated in the last section, that the stationary point is at $\lambda=0^{+}$and $m_{i}=0(i=1,2,3)$. We can also check the nature of this stationary point by evaluating the eigenvalues of the Hessian of $S_{\text {eff }}$, finding that the stationary point (77) is a minimum for $S_{\text {eff }}$ provided $\Lambda_{S}>0$.

\section{A New Source of Decoherence?}

We have speculated that the dynamics of the Universe is not precisely free fall, possibly due to topology-changing absorbtion/emission processes. If so, then in the interval between such interactions the Universe propagates as a virtual particle in superspace. Alternatively, as we have suggested in some previous articles, the massshell constraint may not really be a constraint at the quantum level. In either case, the Universe would be propagating somewhat off-shell. It is interesting to imagine how this off-shell character might manifest itself, if the effect would be large enough to be observable. 
Consider a solution of the evolution equation (33) and constraints $(\delta Æ / \delta \mathcal{N}) \Psi=0$, which is a superposition of two WKB states

$$
\Psi(q, \tau)=\Psi_{A}(q, \tau)+\Psi_{B}(q, \tau)
$$

of the form

$$
\begin{aligned}
& \Psi_{A}(q, \tau)=\int d \mathcal{E} D \alpha F_{A}(\mathcal{E}, \alpha) \exp \left[\frac{i}{\hbar}\left\{\left(\mathcal{E}-\mathcal{M}^{2}\right) \tau-\sqrt{\mathcal{E}} S[Q, \alpha]\right\}\right] \phi_{A}(q) \\
& \Psi_{B}(q, \tau)=\int d \mathcal{E} D \alpha F_{B}(\mathcal{E}, \alpha) \exp \left[\frac{i}{\hbar}\left\{\left(\mathcal{E}-\mathcal{M}^{2}\right) \tau-\sqrt{\mathcal{E}} S[Q, \alpha]\right\}\right] \phi_{B}(q)
\end{aligned}
$$

where $\tau=s / 2 \mathcal{M}$ is the rescaled evolution (proper-time) parameter and $F_{A, B}$ are distributions concentrated at $\mathcal{E}=\mathcal{M}^{2}$ (with a rms uncertainty $\Delta \mathcal{E}$ ) and at parameter values $\{\alpha\}=\left\{\alpha_{A, B}\right\}$ respectively. The functional $S[Q, \alpha]$ is a solution, invariant under 3 -space diffeomorphisms, of the Hamilton-Jacobi equation

$$
\kappa^{2} G^{i j} \frac{\delta S}{\delta Q^{i}(x)} \frac{\delta S}{\delta Q^{j}(x)}+\sqrt{g} \mathcal{U}[Q(x)]=0
$$

with $\{\alpha\}$ a set of integration constants. In these equations $Q$ represents the set of degrees of freedom to be treated semiclassically, and $\sqrt{g} \mathcal{U}[Q]$ is the part of the superpotential involving only those degrees of freedom. Note that in the case of onshell propagation, i.e. $\mathcal{E}=\mathcal{M}^{2}$, the $\tau$-dependence drops out of the wavefunction, and the expressions in (79) are just WKB solutions of the Wheeler-DeWitt equation.

Let us imagine that in some region of superspace where the amplitudes $\Psi_{A, B}$ are non-negligible, the phase difference

$$
\delta S\left[Q^{\prime}\right]=\left|S\left[Q, \alpha_{A}\right]-S\left[Q, \alpha_{B}\right]\right|
$$

depends mainly on a small subset $Q^{\prime}$ of the $Q$ degrees of freedom. For example, $Q^{\prime}$ might refer to the location of a particle recorded on a photographic plate, and $\delta S$ refers to the difference in action, associated with two well separated particle paths in an interferometer, leading to the same final location.

We now ask whether the two components $\Psi_{A}$ and $\Psi_{B}$ will interfere coherently, in the sense that the term is used in optics, in a measurement of $Q^{\prime} \subset Q$. If $\Delta \mathcal{E} \neq 0$, then we must consider stationarity with respect to variation in $\mathcal{E}$, as well as stationarity with respect to variations in the parameters $\alpha$. The stationary phase condition tells us 
that the components $\Psi_{A}$ and $\Psi_{B}$ are peaked at a given configuration $Q$ at parameter times

$$
\tau_{A}=\frac{S\left[Q, \alpha_{A}\right]}{2 \sqrt{\mathcal{E}}} \quad, \quad \tau_{B}=\frac{S\left[Q, \alpha_{B}\right]}{2 \sqrt{\mathcal{E}}}
$$

respectively, with $\mathcal{E}$ evaluated at $\mathcal{E}=\mathcal{M}^{2}$. Interference of wavefunctions $\Psi_{A}$ and $\Psi_{B}$ is coherent, in the sense of physical optics, if the relative phase between the two wavefunctions is constant in the $\tau$-interval $\left[\tau_{A}, \tau_{B}\right]$. In standard terminology the "linewidth" of the wavefunction is $\Delta \mathcal{E} / \hbar$, and the "coherence time" is $\Delta \tau=\hbar / \Delta \mathcal{E}$. If the linewidth has a stochastic origin, then the phase of the wavefunction at $\tau+\Delta \tau$ is not related in a simple way to the phase at parameter time $\tau$. The coherence criterion is then

$$
\delta \tau<\Delta \tau=\frac{\hbar}{\Delta \mathcal{E}}
$$

where

$$
\delta \tau \equiv\left|\tau_{A}-\tau_{B}\right| \approx \frac{1}{2 \sqrt{\mathcal{E}}} \delta S\left[Q^{\prime}\right]
$$

which means

$$
\frac{1}{2 \sqrt{\mathcal{E}}} \delta S<\frac{\hbar}{\Delta \mathcal{E}}
$$

Defining $\hbar_{e f f}(\mathcal{E})=\hbar / \sqrt{\mathcal{E}}$ and the dispersion

$$
\delta \hbar=\left|\frac{d}{d \mathcal{E}} \hbar_{e f f}\right|_{\mathcal{E}=\mathcal{M}^{2}} \Delta \mathcal{E}=\frac{1}{2} \hbar_{e f f} \frac{\Delta \mathcal{E}}{\mathcal{E}}
$$

the condition for coherent interference becomes

$$
\frac{\delta S}{\hbar_{e f f}}<\frac{\hbar_{e f f}}{\delta \hbar}
$$

The argument above is quite general, and applies to any WKB treatment of the evolution equation (33). In fact, if one is prepared to accept that there may be a stochastic uncertainty $\delta \hbar$ (of whatever origin) in the phenomenological value of Planck's constant, then a condition of the form (87) can be easily deduced from the standard Feynman path integral in fixed background spacetime. If there are two or more semiclassical paths which contribute to a given transition amplitude at leading order in $\hbar$, i.e.

$$
G\left[q_{f}, q_{0}\right] \approx \sum_{i} \mu_{i} e^{i S_{i}\left[q_{f}, q_{0}\right] / \hbar}
$$

and if $\hbar$ itself has some dispersion $\delta \hbar$, then the relative phase between path $i$ and path $j$ becomes indeterminate if the inequality (87) is violated, where $\delta S=\left|S_{i}-S_{j}\right|$ is the difference in action of the two paths, and $\hbar_{e f f} \equiv \hbar$. 
A signature of finite dispersion $\delta \hbar$ in the effective value of Planck's constant could be, e.g., an observed decoherence of particle beams in an ultra-sensitive particle interferometer, in a situation where standard time-energy considerations would imply that the beams should interfere coherently. In this case, the wavefunction $\Psi_{A}\left(\Psi_{B}\right)$ represents the contribution to the full "wavefunction of the Universe" $\Psi$ in which the particle travels through path A (B) of the interferometer, respectively, while $\delta S / \hbar_{\text {eff }}$ is a WKB phase difference associated with this difference in path. If the Universe propagates slightly off-shell, as has been suggested here, then the interference will be incoherent if the inequality (87) is violated. To our knowledge no such decoherence has ever been observed, and, in the absence of any theoretical lower bound on $\delta \hbar$, a more detailed discussion of particle interferometry in this context would be premature.

Of course, any finite dispersion in Planck's constant would also feed into finite uncertainties in every other physical quantity, and some of these quantities have been measured quite accurately. In particular, $\hbar / e^{2}$ can be deduced, by combining $g-2$ measurements with high-order QED calculations, to one part in $10^{12}$. However, an ultra-high accuracy measurement of some physical constant, such as $\hbar / e^{2}$, does not necessarily project the Universe into an eigenstate of $\mathcal{E}$ (or $\hbar_{e f f}$ ). Planck's constant is not determined from a single measurement (although $g-2$ can be determined from observations of a single electron), and the reported value would be, in our formalism, an average value for $\hbar_{e f f}$, at the average value $\mathcal{E}=\mathcal{M}^{2}$. For example, in the $g-2$ experiments, one adjusts a rf frequency to maximize the number of spin flips of a trapped electron [10]. Naturally, the peak in spin-flips versus frequency has a certain width. The dispersion $\delta \hbar$, if indeed there is such a dispersion, would be a contribution (perhaps negligible, compared to other sources) to that width, while the center of the peak would locate, in the quantity $\hbar / e^{2}$, only the average value of the effective Planck's constant.

\section{Conclusions}

We have seen that the classical dynamics of bosonic fields (including gravity) in a closed Universe can be re-expressed as describing the free fall of a point particle in superspace. The Hamiltonian operator describing this "particle" contains a (classically unobservable) parameter $\mathcal{M}$ analogous to mass, and the usual Hamiltonian constraint of general relativity can be viewed, in terms of this parameter, as a mass- 
shell condition.

This "free-fall" description of general relativity is, of course, a formal result. Conceivably it also has physical content, and we have suggested two possibilities: First, quantum effects (virtual universe loops) could induce an effective action for the (nonstandard) supermetric, and this action is essentially a function of the coupling constants of the bosonic field theory. In various minisuperspace models, we have seen that the effective action (or at least, the first terms in its adiabatic expansion) is stationary for vanishing cosmological constant. We do not know whether this desirable feature survives in the full theory. Secondly, one may speculate that the universe, propagating like a particle, may propagate slightly off-shell. In principle this could lead to some very interesting effects, as suggested in the last section, but unfortunately we have no estimate to offer of their magnitude.

\section{Acknowledgements}

J.G.'s research is supported in part by the U.S. Dept. of Energy, under Grant No. DE-FG03-92ER40711. A.C.'s research is supported by a JSPS postdoctoral fellowship, under contract No. P95196; he would like to thank the cosmology group at Tokyo Institute of Technology for the kind hospitality during this work. Support was also provided by the Director, Office of Energy Research, Office of Basic Energy Services, of the U.S. Department of Energy under Contract DE-AC03-76SF00098. 


\section{A $\quad N_{s}=1$ massive scalar field}

In the case of a single massive, minimally coupled scalar field in a FRW geometry, the effective action (44) (neglecting contributions coming from the supercurvature terms) reads

$$
\begin{aligned}
S_{e f f} & \simeq \Lambda_{S} \int_{0}^{\bar{a}} d a \int_{-\phi_{M}}^{\phi_{M}} d \phi a^{3}\left|\left(m^{2} \phi^{2}+\lambda\right) a^{2}-1\right| \\
& =\frac{\Lambda_{S} I_{\phi} \bar{a}^{4}}{36 x}\left\{[2(3 x+y)-9] x+\frac{3}{(x+y)}\right. \\
& +\Theta(x) \frac{3}{(x y)^{1 / 2}} \arcsin \left[\frac{y^{1 / 2}}{(x+y)^{1 / 2}}\right] \\
& -\Theta(-x)\left[\frac{(1-x)^{1 / 2}\left(8 x^{2}-14 x+3\right)}{y^{1 / 2}}\right. \\
& \left.\left.+\frac{3}{(-x y)^{1 / 2}} \ln \left[\frac{\left[y^{1 / 2}+(-x)^{1 / 2}\right]^{1 / 2}}{\left[y^{1 / 2}-(-x)^{1 / 2}\right]^{1 / 2}\left[(-x)^{1 / 2}+(1-x)^{1 / 2}\right]}\right]\right]\right\}
\end{aligned}
$$

where, in performing the integrations in $a$ and $\phi$, we have assumed that the cutoff regulators satisfy the conditions $|\lambda| \bar{a}^{2}>1$ and $m^{2} \phi_{M}^{2}>|\lambda|$, and we have defined, as usual, the variables $x$ and $y$ according to eqs. (51) and (72). Taking the partial derivatives of $S_{\text {eff }}$ with respect to $\lambda$ and $m^{2}$ we then get (for $x>0$ )

$$
\begin{aligned}
\frac{\partial S_{\text {eff }}}{\partial \lambda} & \simeq \frac{\Lambda_{S} I_{\phi} \bar{a}^{6}}{24}\left\{\frac{\left[4(x+y)^{2} x^{2}-(5 x+3 y)\right]}{(x+y)^{2}}\right. \\
& \left.-\frac{3}{(x y)^{1 / 2}} \arcsin \left[\frac{y^{1 / 2}}{(x+y)^{1 / 2}}\right]\right\} \\
\frac{\partial S_{\text {eff }}}{\partial\left(m^{2}\right)} & \simeq \frac{\Lambda_{S}\left(I_{\phi}\right)^{3} \bar{a}^{6}}{288 x y}\left\{\frac{\left[4 x y(x+y)^{2}+3(x-y)\right]}{(x+y)^{2}}\right. \\
& \left.-\frac{3}{(x y)^{1 / 2}} \arcsin \left[\frac{y^{1 / 2}}{(x+y)^{1 / 2}}\right]\right\}
\end{aligned}
$$

Summing and subtracting eqs. (90), it is easy to check that the stationarity conditions for the effective action become (for $x>0$ )

$$
\begin{aligned}
2 & \simeq(x+y)^{2}(x-y) \\
\frac{y}{x+y} & \simeq \sin ^{2}\left[\frac{(x y)^{1 / 2}\left(3 x^{2}+3 y^{2}+2 x y\right)}{6}\right]
\end{aligned}
$$

The method is to solve the first of conditions (91) for $x$ in terms of $y$, and then to solve for $y$ from the second of (91). Although the functional dependence of $x$ on $y$ 
is unique, it is easy to check that, already when $x>0$, there is an infinite set of stationary points $y_{n}$, with $x_{n}\left(y_{n}\right) \sim \beta y_{n} \rightarrow+\infty$ as $n \rightarrow \infty$. In other words, the stationary points of $S_{\text {eff }}$ for $x>0$ will be at

$$
\begin{aligned}
\lambda_{n} & \sim \frac{\beta M_{n}}{\bar{a}^{2}} \\
m_{n}^{2} & \sim \frac{M_{n}}{\phi_{M}^{2} \bar{a}^{2}}
\end{aligned}
$$

Unfortunately, since $M_{n} \rightarrow \infty$ as $n \rightarrow \infty$, one cannot make any reliable prediction (unless one assumes some - unnatural - scaling between $M_{n}$ and the cutoff $\bar{a}$ ) about the values of $\lambda$ and $m$ in this toy model. 


\section{References}

[1] R. P. Feynman, Phys. Rev. 80 (1950) 440.

[2] E. Stueckelberg, Helv. Phys. Acta 14 (1941) 332; 588;

V. Fock, Phys. Z. Sowjetunion 12 (1937) 404;

Y. Nambu, Prog. Theor. Phys. 5 (1950) 82;

J. Schwinger, Phys. Rev. 82 (1951) 664.

[3] J. Greensite, Class. Quantum Grav. 13 (1996) 1339.

[4] B. S. DeWitt, in Relativity, ed. M. Carmeli, S. Fickler, and L. Witten (Plenum, New York, 1970).

[5] R.F. Baierlein, D.H. Sharp, and J.A. Wheeler, Phys. Rev. 126 (1962) 1864;

J. A. Wheeler, in Relativity, Groups, and Topology, ed. C. DeWitt and B. DeWitt (Gordon and Breach, New York, 1964).

[6] A. Carlini and J. Greensite, Phys. Rev. D52 (1995) 936.

[7] A. Carlini and J. Greensite, Phys. Rev. D52 (1995) 6947.

[8] S. Giddings and A. Strominger, Nucl. Phys. B321 (1989) 481;

V. Rubakov, Phys. Lett. B214 (1988) 503;

M. McGuigan, Phys. Rev. D38 (1988) 3031.

[9] S. Coleman, Nucl. Phys. B310 (1988) 668.

[10] R. Van Dyck Jr., P. Schwinberg, and H. Dehmelt, Phys. Rev. D34 (1986) 722. 